\title{
Cancer-immune interactions in ER-positive breast cancers: PI3K pathway alterations and tumor-infiltrating lymphocytes
}

Marcelo Sobral-Leite ${ }^{1,2}$, Izhar Salomon ${ }^{1}$, Mark Opdam ${ }^{1}$, Dinja T. Kruger ${ }^{1,6}$, Karin J. Beelen ${ }^{1,8}$, Vincent van der Noort ${ }^{7}$, Ronald L. P. van Vlierberghe ${ }^{11}$, Erik J. Blok ${ }^{11,12}$, Daniele Giardiello ${ }^{1}$, Joyce Sanders ${ }^{3}$, Koen Van de Vijver ${ }^{4,5}$, Hugo M. Horlings ${ }^{3}$, Peter J. K. Kuppen ${ }^{11}$, Sabine C. Linn ${ }^{1,9,10}$, Marjanka K. Schmidt ${ }^{1}$ and Marleen Kok K $^{9,13^{*}}$

\begin{abstract}
Introduction: The presence of tumor-infiltrating lymphocytes (TILs) is correlated with good prognosis and outcome after (immuno)therapy in triple-negative and HER2-positive breast cancer. However, the role of TILs in luminal breast cancer is less clear. Emerging evidence has now demonstrated that genetic aberrations in malignant cells influence the immune landscape of tumors. Phosphatidylinositol 3-kinase (PI3K) is the most common altered pathway in ER-positive breast cancer. It is unknown whether changes in the PI3K pathway result in a different composition of the breast tumor microenvironment. Here we present the retrospective analysis of a prospective randomized trial in ER-positive breast cancer on the prognostic and predictive value of specific tumor-associated lymphocytes in the context of PI3K alterations.

Methods: We included 563 ER-positive tumors from a multicenter trial for stage I to III postmenopausal breast cancer patients, who were randomized to tamoxifen or no adjuvant therapy. The amount of CD8-, CD4-, and FOXP3-positive cells was evaluated by immunohistochemistry and quantified by imaging-analysis software. We analyzed the associations between PIK3CA hotspot mutations, PTEN expression, phosphorylated proteins of the PI3K and MAPK pathway (p-AKT, p-ERK1/2, p-4EBP1, p-p70S6K), and recurrence-free interval after adjuvant tamoxifen or no adjuvant treatment.

Results: CD8-positive lymphocytes were significantly more abundant in PIK3CA-mutated tumors (OR $=1.65 ; 95 \% \mathrm{Cl}$ 1.03-2.68). While CD4 and FOXP3 were not significantly associated with prognosis, patients with tumors classified as CD8-high had increased risk of recurrence $(H R=1.98 ; 95 \% \mathrm{Cl} 1.14-3.41$; multivariable model including PIK3CA status, treatment arm, and other standard clinicopathological variables). Lymphocytes were more often present in tumors with increased PI3K downstream phosphorylation. This was most pronounced for FOXP3-positive cells.

Conclusion: These exploratory analyses of a prospective trial in luminal breast cancer suggest high CD8 infiltration is associated with unfavorable outcome and that PI3K pathway alterations might be associated with the composition of the tumor microenvironment.
\end{abstract}

Keywords: Luminal breast cancer, Tumor-infiltrating lymphocytes, PIK3CA mutations, PI3K pathway

\footnotetext{
*Correspondence: m.kok@nki.nl

${ }^{9}$ Division of Medical Oncology, Netherlands Cancer Institute, Amsterdam, The

Netherlands

${ }^{13}$ Division of Molecular Oncology and Immunology, Netherlands Cancer

Institute, Plesmanlaan 121, 1066 CX Amsterdam, The Netherlands

Full list of author information is available at the end of the article
}

(c) The Author(s). 2019 Open Access This article is distributed under the terms of the Creative Commons Attribution 4.0 International License (http://creativecommons.org/licenses/by/4.0/), which permits unrestricted use, distribution, and reproduction in any medium, provided you give appropriate credit to the original author(s) and the source, provide a link to the Creative Commons license, and indicate if changes were made. The Creative Commons Public Domain Dedication waiver (http://creativecommons.org/publicdomain/zero/1.0/) applies to the data made available in this article, unless otherwise stated. 


\section{Introduction}

The prognosis of early luminal breast cancer patients has substantially improved after the introduction of endocrine therapy (tamoxifen and aromatase inhibitors) [1]. Even so, around $20 \%$ of patients still experience recurrence of disease [2]. Accumulating evidence including multiple independent studies and meta-analyses have shown the correlation between tumor-infiltrating lymphocytes (TILs) and favorable prognosis in triple-negative breast cancer (TNBC) and human epidermal growth factor receptor 2 (HER2)-positive breast cancer [3, 4]. The prognostic value of TILs in ER-positive/HER2-negative disease remains relatively unclear, and conflicting results have been reported [3, 5-13]. There is no solid biological explanation for this different role of TILs in ER-positive/ HER2-negative breast cancer. Recently, emerging experimental and clinical studies have demonstrated that genetic aberrations in cancer cells can influence the immune landscape of tumors [14-18]. Common drivers of tumorigenesis, such as but not limited to p53, Notch, and MYC, can modulate immune signaling pathways [14-18]. Studying the relationship between the genetic make-up of luminal breast carcinomas and the infiltration of specific subsets of immune cells is crucial for the rational design of clinical trials evaluating the combination of novel targeted therapy with immunomodulatory agents.

The phosphatidylinositol 3-kinase (PI3K) pathway is one of the most altered pathways in luminal breast cancer, and large efforts to unravel endocrine resistance have pointed to aberrant signaling of PI3K, e.g., by PIK3CA mutations, loss of PTEN, or downstream protein phosphorylation [19-23]. It led to the development of treatment strategies like mTOR and PI3K inhibitors [19, 21, 24, 25]. Adding PI3K inhibitors to standard endocrine treatment results in improvement of progressionfree survival (PFS) in patients with metastatic disease with a PIK3CA-mutated tumor [25]. So far, it is largely unknown whether PI3K alterations influence the composition of the tumor microenvironment.

Another treatment strategy that is currently under investigation in luminal breast cancer includes immune checkpoint blockade. Although first results suggest that response rates are modest, durable responses have been observed in luminal metastatic breast cancer [26]. For further improvement of immunomodulatory treatments for luminal breast cancers, evaluating the presence of specific immune cell subsets as well as a better understanding of the cancer-immune interactions will be crucial.

$\mathrm{T}$ lymphocytes are involved in the cytotoxic adaptive immunity and play a central role in antitumor immune responses [27, 28]. Different subpopulations of T lymphocytes regulate the balance of immune tolerance and proinflammatory status in tumors [17, 28-30]: CD8-positive cytotoxic $\mathrm{T}$ lymphocytes are involved in interferon gamma
(IFN $\gamma$ )-dependent mechanisms of antitumor activity $[11,28,29]$; CD4-positive helper T lymphocytes are important in activating and regulating the adaptive immune response [28, 30]; in contrast, FOXP3-positive regulatory $\mathrm{T}$ lymphocytes have a crucial role in protecting tissues from inflammation damage and preventing autoimmunity $[13,30]$. In the tumor microenvironment, regulatory $\mathrm{T}$ lymphocytes play key roles in inhibiting antitumor immune responses [29, 31].

A deeper understanding of the prognostic role of these TIL subsets, their predictive value for endocrine treatment benefit, and the relationship between the TIL subsets and PI3K activity will be useful for future trial design. It will help to find the optimal patient population for the evaluation of immunotherapy and/or PI3K inhibitors in ER-positive breast cancer. Here we present results of analyses on the prognostic and predictive value of specific tumor-associated lymphocytes in the context of PI3K alterations, using data from a trial in which breast cancer patients were randomized between adjuvant tamoxifen and no endocrine treatment.

\section{Materials and methods \\ Study population and samples}

The IKA trial recruited stage I to III postmenopausal breast cancer patients between 1982 and 1994. Patients were randomized $(2: 1)$ between 1 year of tamoxifen (30 mg per day) and no adjuvant therapy. After 1 year, a second randomization was performed between another 2 years of tamoxifen or to stop further treatment. None of the patients received chemotherapy. The study data were part of an Oxford systematic review that evaluated the effect of tamoxifen and were previously analyzed to study and validate biomarkers for tamoxifen resistance [1, 22, 23, 32-34]. Of 1662 patients included, we were able to retrieve 739 formalin-fixed paraffin-embedded (FFPE) tumor blocks. Tissue microarrays (TMAs) were constructed using three cores $(0.6 \mathrm{~mm}$ each) per tumor. Of those tumors, 563 were ER-positive (ER $\alpha$ positivity defined as $\geq 10 \%$ positive tumor cells as determined using immunohistochemistry) [22, 23] (Additional file 1: Figure S1). Data collection of patient characteristics, including the pathology variables, expression of progesterone receptor (PR), HER2, and clinical outcome, was described previously [22, 23]. Ki67 staining was evaluated by the percentage of nuclear positive tumor cells (Additional file 2: Table S1). The original trial was approved by the central ethics committee of the Netherlands Cancer Institute (NKI), and informed consent was obtained from all study participants.

\section{PIK3CA mutation status}

DNA isolated from FFPE tumor blocks was genotyped using Sequenom mass spectrometry-based genotyping technology to assess the hotspot mutations of the PIK3CA 
gene: in exon 9, 1624G>A (E542K) and 1633G >A (E545K), and exon 20, 3140A $>\mathrm{T}(\mathrm{H} 1047 \mathrm{~L})$ and 3140A $>\mathrm{G}(\mathrm{H} 1047 \mathrm{R})$, as described previously [23]. Genotyping for PIK3CA exon 9 mutations was successful in 488 tumor samples, while exon 20 mutations could be assessed in 491 tumor samples [23] (Additional file 1: Figure S1).

\section{Evaluation of PI3K pathway activity}

Phosphorylation status of proteins from the PI3K pathway: p-AKT (Thr308 and 473), p-4EBP1 (Ser65), and pp70S6K (Thr389); from the MAPK pathway: p-ERK1/2 (Thr202/Tyr204); and PTEN expression were assessed using immunohistochemistry (IHC) on TMAs. The details on the IHC analyses and the criteria used for scoring of these markers were previously reported and are summarized in Additional file 2: Table S1 [22, 23, 33, 34].

\section{Evaluation of the lymphocyte markers}

Whole serial sections of 563 ER-positive FFPE tissue blocks were stained for CD4, CD8, and FOXP3. IHC was performed using the Ventana Benchmark ${ }^{\circ}$ Ultra system (Ventana Medical Systems, Tucson, USA), and details are provided in Additional file 2: Table S1. The slides stained for CD8 and CD4 were scanned using the Aperio Scanner (Leica Biosystems, San Diego, CA, USA) and automatically evaluated by image-analysis software (Tissue Image Analysis, version 2.0, Slidepath, Leica Biosystems, Milton Keynes, UK). The Slidepath software was manually trained to identify and quantify cells in the image of the scanned tumor sections according to staining color, size, and shape of the cells, similar as previously reported [35]. Based on this data, the software calculated the percentage of positive CD4- and CD8-positive lymphocytes among all cells of the tumor tissue section. Slides stained for FOXP3 were scanned using the Philips Ultra Fast Scanner 1.6 RA. FOXP3 nuclear positivity of lymphocytes in intratumoral and in surrounding stroma tissue was automated measured by AxioVision 4.6 (Carl Zeiss Vision, Jena, Germany), as previously described [8]: four fields per slide (in average $1.5 \mathrm{~mm}^{2}$ per field) were used to count the number of FOXP3-positive cells per $\mathrm{mm}^{2}$.

Since the distribution of the expression values of these markers showed lognormal properties, we took the logarithm of these values and centered the resulting distributions in zero by subtracting the logarithmic median of each marker. Two certified pathologists ( $\mathrm{HH}$ and KVdV) and a molecular biologist (Iz) scored the percentage of CD4-, CD8-, and FOXP3-positive lymphocytes in a selected number of cases (HH: $n=33$, KVdV: $n=30$, and Iz: $n=100)$. Samples quantified as zero in the automated and normalized expression correspond, approximately, to the following pathologists' scores: 5\% CD4-, 15\% CD8-, and 1\% FOXP3-positive cells, respectively (Additional file 1: Figure S2). From the 563 ER-positive breast cancer patients included in the trial, 161 to 185 tumor samples had insufficient tumor material to evaluate the expression of these markers. We could quantify CD4, CD8, and FOXP3 expression in 396, 402, and 378 tumor samples, respectively (Additional file 1: Figure $\mathrm{S} 1$ ).

\section{Statistical analyses}

Associations were evaluated by the chi-square test or by Fisher exact test if one of the expected counts were less than 5. Comparison between pathologists' and the expression of lymphocyte markers obtained by automated fashion was calculated by Spearman's rank correlation test and by the intraclass correlation coefficient (ICC; assuming two-way random single measures) [36]. Comparisons of means between two or more groups were examined by Student's $t$ test or ANOVA, respectively. Odds ratios (OR) with their respective 95\% confidence intervals $(95 \% \mathrm{CI})$ were calculated using binomial logistic multivariable regression models to measure the association between lymphocyte markers and PIK3CA mutation status. Associations between tumor variables and lymphocytes or (phospho-) protein levels were calculated by linear regression models and quantified in regression coefficients $(r)$ and their $95 \% \mathrm{CI}$.

Survival time was calculated from the date of randomization. End of follow-up was defined as the date of an event, lost to follow-up, or 10 years time at risk (censoring), whatever came first. The recurrence-free interval (RFI) was defined as the time from the date of first randomization until the occurrence of a local, regional, or distant recurrence, or breast cancer-specific death [22]. Breast cancer-specific interval (BCSI) was defined as death caused by breast cancer progression or a breast cancer treatment-related event. Secondary primary breast tumor was not considered an event, and these patients were censored at the date of this occurrence [22].

Hazard ratios (HRs) and 95\% CI were estimated using Cox regression models. Of note, after 1989, two interim analyses showed a significant improvement in RFI in lymph node (LN)-positive patients. After this year, LNpositive patients in this trial skipped the first randomization and all received 1 year of tamoxifen [32]. Therefore, multivariable Cox regression models were stratified by LN status. Regression analysis included the following variables in the (logistic, linear, and Cox) multivariable models: morphology type (ductal, lobular, or others), tumor grade (1,2, or 3 ), tumor size $(\leq 2 \mathrm{~cm}$ or $>2 \mathrm{~cm}$ ), PR (negative or positive), HER2 (negative or positive), and PIK3CA mutation status (wild-type or mutated). Cox regression models also included age at diagnosis (as continuous value) and tamoxifen arm (treated or control). Splined curves of the Cox regression terms against the expression of lymphocyte markers were used to check for non-linear associations (degree of 
freedom =3) [37]. Cut-off estimations for the lymphocyte markers (low or high) were based on the best sensitivity and specificity measures for RFI prediction [38]. We used a freely available web application to calculate the threshold (R package: Optimal.cutpoints). The method (MaxKappa) makes full use of the clinical and molecular information to assess the prediction value over chance [39, 40].

Unsupervised hierarchical clustering and Euclidean dissimilarity was conducted on phosphorylation levels and protein expression values, centered and proportionally scaled. Results of regression models were obtained using patients with complete data only.

All $p$ values reported are from two-sided tests, and the threshold for significance was set at $p=0.05$. No correction for multiple testing was applied. All statistical analyses were performed using RStudio version 3.2.3 (RStudio Team, 2015).

\section{Results}

CD4, CD8, and FOXP3 expression and standard clinicopathological features

Cells positively stained for CD4, CD8, and FOXP3 were mainly found in the stromal areas (Additional file 1: Figure S3A-F). The average scores from two pathologists were compared with the expression values obtained by image-analysis software $\left(r_{\mathrm{CD} 4}=0.752, r_{\mathrm{CD} 8}=0.796\right.$, and $r_{\mathrm{FOXP} 3}=0.836$; Additional file 1: Figure S3G-I). The presence of CD4-, CD8-, and FOXP3-positive cells was strongly correlated $\left(r_{\mathrm{CD} 8 / \mathrm{FOXP} 3}=0.576, r_{\mathrm{FOXP} 3 / \mathrm{CD} 4}=0.503\right.$, and $r_{\mathrm{CD} 4 / \mathrm{CD} 8}=0.652$; Fig. $1 \mathrm{a}-\mathrm{c}$ ).

Figure $1 \mathrm{~d}-\mathrm{f}$ shows the correlation between CD4-, CD8-, and FOXP3-positive cells and clinicopathological characteristics as calculated by multivariable linear regression. More CD8-, CD4-, and FOXP3-positive cells were detected in high-grade tumors (grade 3) (all $p<0.001$; univariable analyses in Additional file 1: Figure S4A-C). In line with these associations, tumors with high Ki67 scores were associated with higher levels of CD4, CD8, and FOXP3 $(p<0.001$; Additional file 1: Figure S4D-F). HER2-positive tumors showed higher CD8 expression compared with HER2negative tumors $\left(r_{\mathrm{CD} 8}=0.36, p=0.040\right.$; Fig. 1e). FOXP3 expression was significantly lower in large tumors $(>2 \mathrm{~cm}$; $\left.r_{\mathrm{FOXP} 3}=-0.38, p=0.018\right)$ and in lobular carcinomas $\left(r_{\mathrm{FOXP} 3}=-0.59, p=0.015 ;\right.$ Fig. 1f $)$, compared with ductal carcinomas $(p<0.001$; Additional file 1: Figure S4I).

\section{CD4, CD8, and FOXP3 expression and PIK3CA mutations}

PIK3CA mutations in exon 9 or exon 20 were found in 159 (32.7\%) of the 486 tumors genotyped [23]. In total, 296 patients had complete information for PIK3CA mutation and the three immune markers. The tumor microenvironment of PIK3CA-mutated tumors was infiltrated with relatively more CD8-positive cells compared to wild-type tumors ( $p=0.038$, Fig. 2a, b). Linear multivariable regression models showed a relatively weak but statistically significant correlation between PIK3CA mutation status and higher CD4 and CD8 levels $\left(r_{\mathrm{CD} 4}=0.25, p=0.030\right.$ and $r_{\mathrm{CD} 8}=0.27, p=0.005$; Fig. 1d, e).

Two specific PIK3CA mutations were associated with CD8 infiltration: tumors harboring $1633 \mathrm{G}>\mathrm{A}$ mutations in exon 9 or $3140 \mathrm{~A}>\mathrm{G}$ in exon 20 showed slightly higher CD8 expression compared with the wild-type tumors $\left(p_{1633 \mathrm{G}>\mathrm{A}}=0.0473\right.$; Additional file 1 : Figure $\mathrm{S} 5 \mathrm{~B}$ and $p_{3140 \mathrm{~A}>\mathrm{G}}=0.0322$; Additional file 1: Figure S5E).

As previously reported by our group and others [23, 41], low-grade tumors (grade 1 or 2) are likely to have more PIK3CA mutations $\left(\mathrm{OR}_{\text {grade } 3 \mathrm{vs} 1}=0.18\right.$; 95\% CI $0.08-0.38$; Fig. 2a). Within these low-grade tumors, the correlation between CD8 levels and PIK3CA mutation status was more pronounced ( $p=0.001$; Fig. $2 c$ ).

CD4, CD8, and FOXP3 expression and breast cancer outcome Continuous CD4, CD8, and FOXP3 expression levels were not significantly associated with RFI in the Cox linear regression models (data not shown). To illustrate this, we plotted the partial residuals of the (univariate and multivariable) Cox regression models for each lymphocyte marker (Additional file 1: Figure S6A-F). However, it is known that continuous variables can be associated with the hazard in a non-linear way [42]. The spline function of the fitted partial residual values of the Cox regression models for each lymphocyte marker indeed showed a non-linear shape (Additional file 1: Figure S6G-I). Since there is no established cut-off for CD4, CD8, and FOXP3 available yet, these spline plots were used to identify the best cut-off points in the scale of the expression of the lymphocyte markers according to the largest differences in the recurrence rates (Additional file 1: Figure S6J-L). The splined curves highlighted the increase of partial residual values of patients classified as high CD4, CD8, and FOXP3 (Fig. 3a-c). The Kaplan-Meier curves illustrate that patients with tumors harboring high levels of CD8 (29 of the total of 410 tumors) have a worse RFI compared to patients with low CD8 tumors (log-rank test; $p<$ 0.001; Fig. 3e). After multivariable adjustment for PIK3CA mutation and other pathological variables, this association remained significant: $\mathrm{HR}_{\text {high-CD8 }}=1.98 ; 95 \%$ CI $1.14-3.41$ (Fig. 3e and Additional file 1: Figure S7A). Associations between RFI and CD4 and FOXP3 expression were not statistically significant after multivariable analysis (Fig. 3d, f, and Additional file 1: Figure S7A). After exclusion of the patients with HER2-positive disease, we still observe a significantly worse RFI in patients with high CD8 expression in the tumor microenvironment $\left(\mathrm{HR}_{\text {high-CD8 }}=1.87 ; 95 \% \mathrm{CI}\right.$ 1.07-3.28, $p<0.0001$ ).

While ER-positive breast tumors with low grade are considered to have a relative good prognosis in breast cancer [1], within these low-grade tumors, we observed 


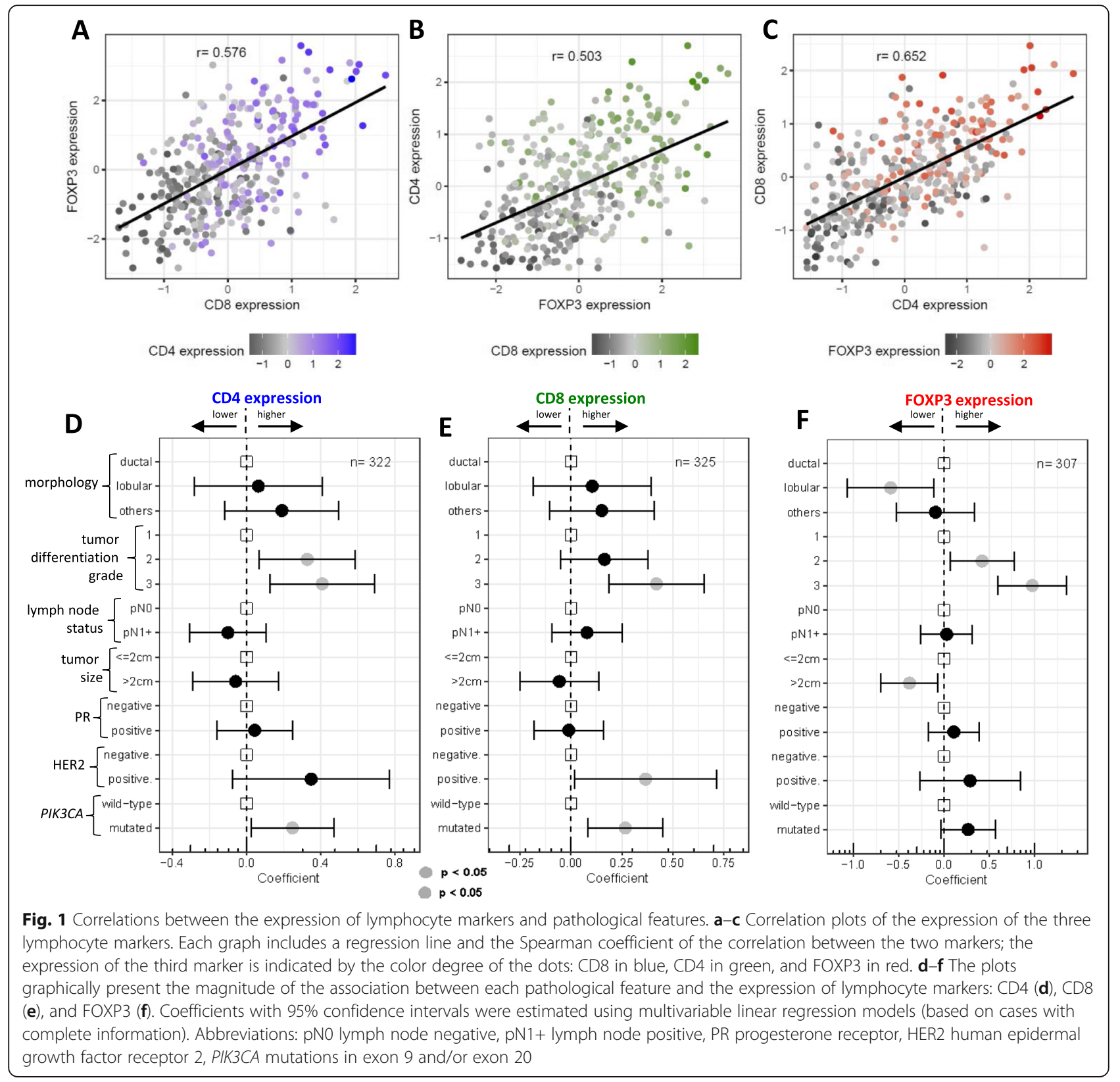

that high levels of CD4 or CD8 were associated with poor outcome (log-rank test; $p<0.0001$; Fig. 3g, h). The effect remained significant after adjustment for known prognostic factors (Fig. $3 \mathrm{~g}, \mathrm{~h}$, and Additional file 1: Figure S7B). Similar associations were observed when using BCSI as endpoint (Additional file 1: Figure S7C and D).

\section{CD8 expression and breast cancer outcome according to tamoxifen treatment}

The proportion of patients identified with CD8-high tumors corresponded to only $7.2 \%$ of total tumors quantified $(n=410$; Fig. 4a). We evaluated the prognostic value of CD8 status within each arm of the trial: not-treated and treated with tamoxifen ( 1 or 3 years after surgery). High CD8 levels were associated with poor RFI regardless of tamoxifen treatment (log-rank test; $p<0.001$ and $p=0.040$, respectively; Fig. $4 \mathrm{~b}, \mathrm{c}$ ). However, univariate HR remained significant only for the control arms. The $p$ value for interaction between CD8 status and tamoxifen treatment in a full model for RFI was 0.082 . Since patients with HER2-positive tumors did not receive trastuzumab, we applied the same analysis in the HER2negative patients only. The proportion of CD8-high tumors and RFI associations observed in this subgroup $(n=355)$ were similar to whole ER-positive group. 


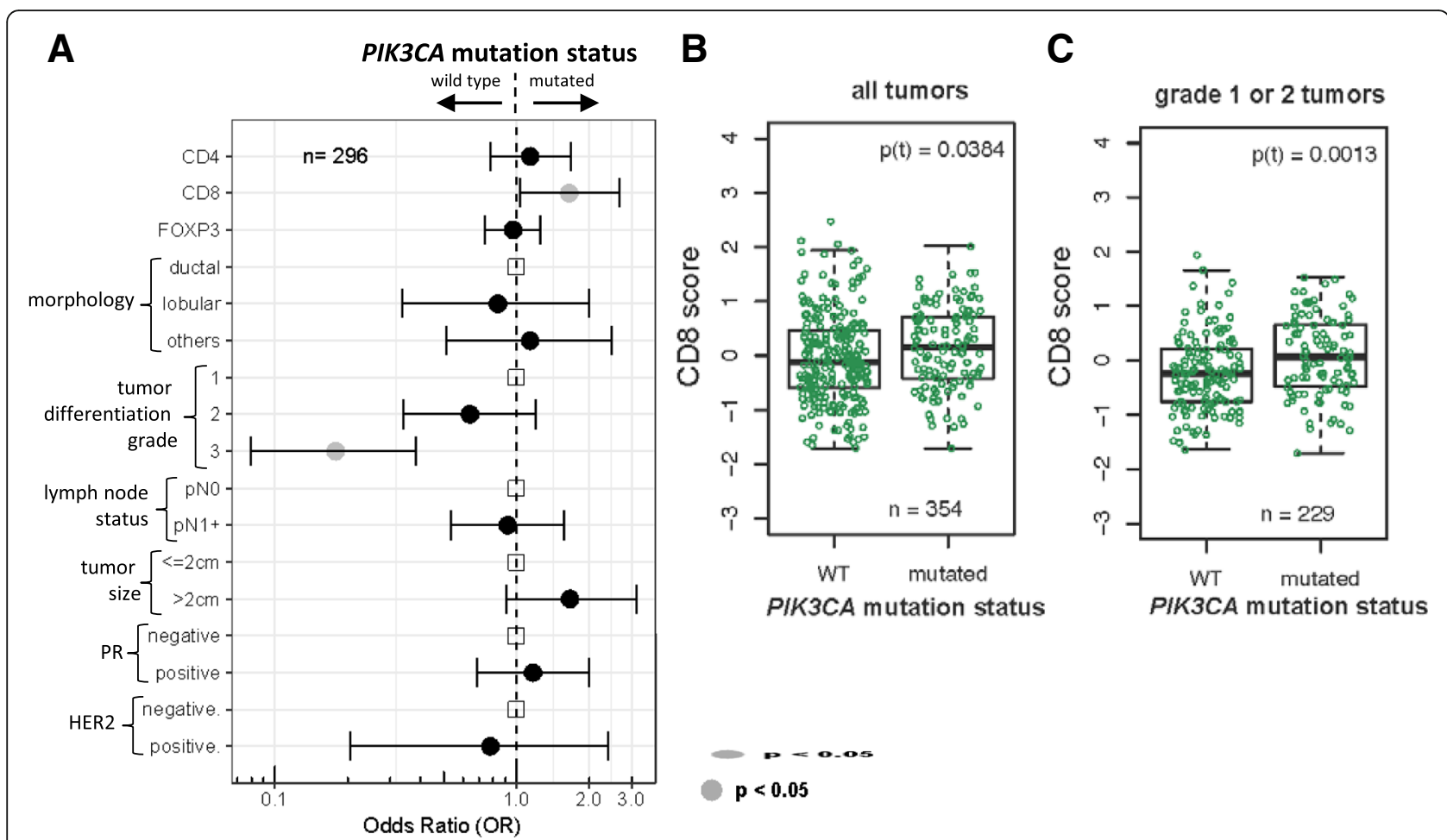

Fig. 2 Expression of the lymphocyte markers and PIK3CA mutation status. a Forest plot graphically represents the magnitude of the association between each pathological feature and PIK3CA mutation status, calculated by multivariable logistic regression model (as complete case analysis). b Distribution of CD8 expression according to PIK3CA mutation status in all tumors and $\mathbf{c}$ within grade 1 or 2 tumors. Statistical differences between the expression means among the two categories were calculated by $t$ test: $p(t)$. Abbreviation: pNo lymph node negative, pN1+ lymph node positive, PR progesterone receptor

Of note, after the first interim analyses of the IKA trial, it was decided that LN-positive patients would skip the first randomization. After this point, all LN-positive patients received 1 year of tamoxifen [32]. Thus, we calculated the effect of adjuvant tamoxifen during 1 or 3 years according to CD8 status, by applying regression analysis grouped by LN status, but it did not change our conclusions (Additional file 1: Figures S8, S9, and Additional file 2: Table S2).

\section{CD4, CD8, and FOXP3 expression and the activation of PI3K pathway in breast tumors}

Unsupervised hierarchical clustering revealed that tumors with increased levels of CD4, CD8, and FOXP3 also had higher phosphorylation levels of proteins from the PI3K pathway (right side of dendrogram and heat map, Fig. 5a).

In order to statistically evaluate this link between the specific phosphorylation of the PI3K pathway and the presence of lymphocytes, we performed multivariable linear regression models including pathological variables and PIK3CA mutation status (Additional file 2: Tables S3A-F). In general, we found a weak positive correlation between the phosphorylation levels of PI3K pathway and lymphocyte infiltration. More specifically, p-AKT(Ser473),
p-ERK1/2, and p-p70S6K were statistically significant associated with high CD4 $(p=0.045,0.003$, and 0.007 , respectively; Fig. 5b). A similar pattern was observed for the associations with CD8, in which p-AKT(Thr308), p$\mathrm{AKT}($ Ser473), and p-p70S6K reached statistical significance $(p=0.030,0.009$, and 0.004, respectively; Fig. 5c). Phosphorylation levels of the PI3K markers and PTEN expression were all positively and significantly correlated with FOXP3 expression (almost all $p<0.001$, Fig. $5 \mathrm{~d}$ and Additional file 2: Tables S3A-F). Exclusion of the HER2positive cases did not substantially change the results (data not shown).

Taking together, ER-positive breast tumors with high levels of TILs show more activation of downstream proteins of the PI3K pathway.

\section{Discussion}

We performed post hoc analysis of TIL composition and PI3K alterations in a trial that compared the outcome of ER-positive breast cancer patients, randomized between adjuvant tamoxifen (1-3 years), and patients who received no adjuvant treatment. For CD4 and FOXP3 expression, we did not find an association with outcome. In contrast, our analyses in the total cohort of ER-positive tumors as well as in the ER-positive/HER2-negative 


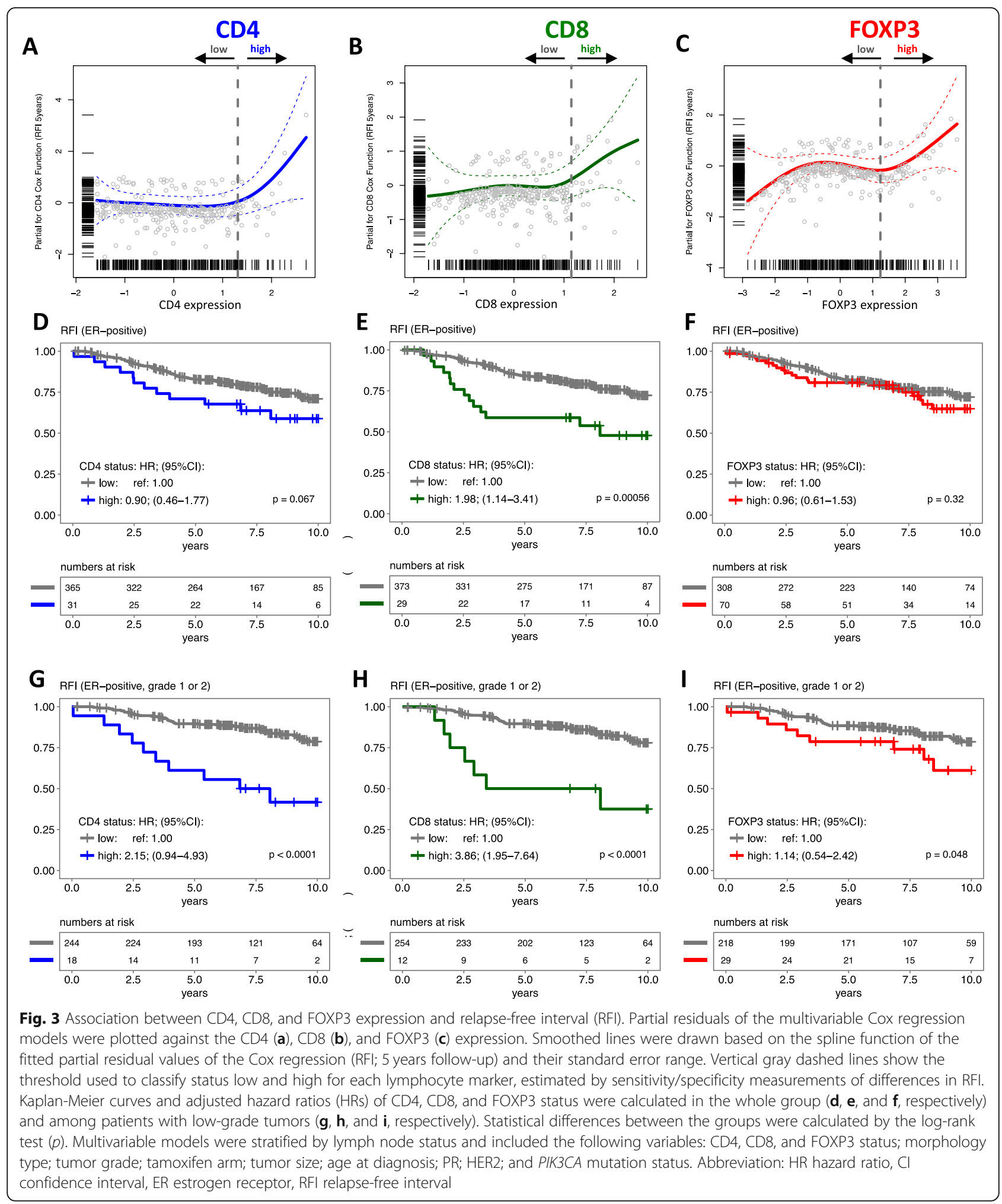

subgroup suggest that patients with ER-positive tumors with very high infiltration of CD8-positive lymphocytes are more likely to have recurrence of disease. This association is in the opposite direction of the established link observed between high TILs and improved prognosis in TN and HER2-positive breast cancer $[4,5,7,11]$. But in the largest observational study so far on CD8, Ali et al. described a relatively poor outcome for CD8-high tumors 


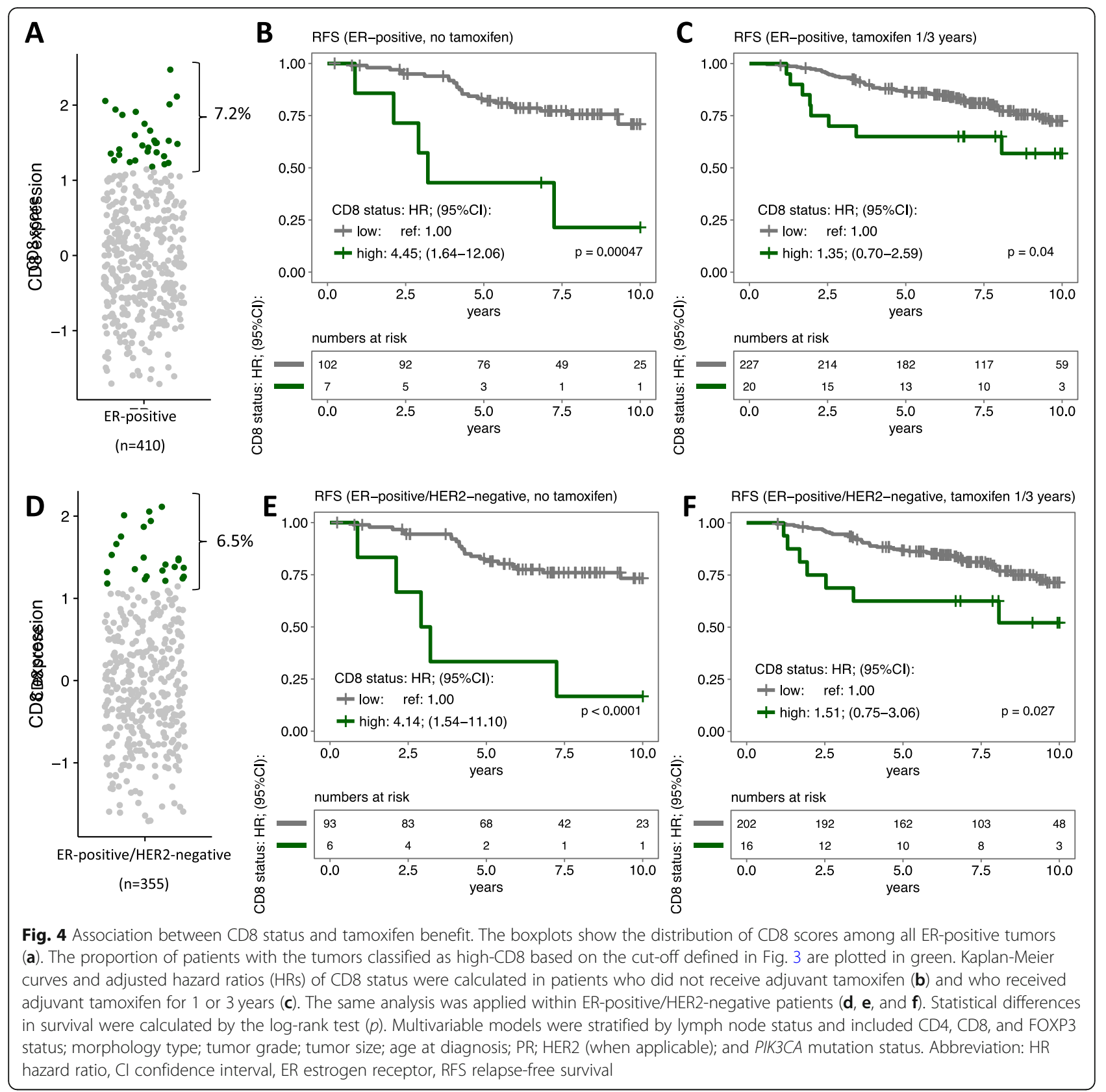

within the ER-positive/HER2-negative subgroup [5]. In addition, our exploratory data is also in line with a recent meta-analysis showing that an increase in TILs is associated with shorter overall survival in luminal-HER2-negative tumors $(\mathrm{HR}=1.10$; 95\% CI 1.02-1.19, $p=0.011)$ [7]. Even though we determined CD8-high threshold using the survival data of this series, which resulted in overfitting, these large studies support the link we found between high CD8 expression and poor survival in luminal breast cancer. A clear explanation for this opposite effect of TIL in ER-positive disease versus patterns seen in TN or HER2-positive breast cancer is still lacking. We checked if tumors with high levels of CD8- positive lymphocytes would also be the ones expressing a lower percentage of ER positivity but no association was found (Additional file 1: Figure S10).

FOXP3 expression did not show significant prognostic value, similar to results from Ali et al. [5], Mahmoud et al. [12], and Baker et al. [6]. However, other studies have reported statistically significant associations between FOXP3+ and poor survival in breast cancer [9, 13]. Although FOXP3 status alone was not predictive for recurrence risk in our data, its expression was highly correlated with CD4 and CD8 expression. However, scoring of FOXP3-positive lymphocytes remains challenging for pathologists since these cells are often scattered in the 


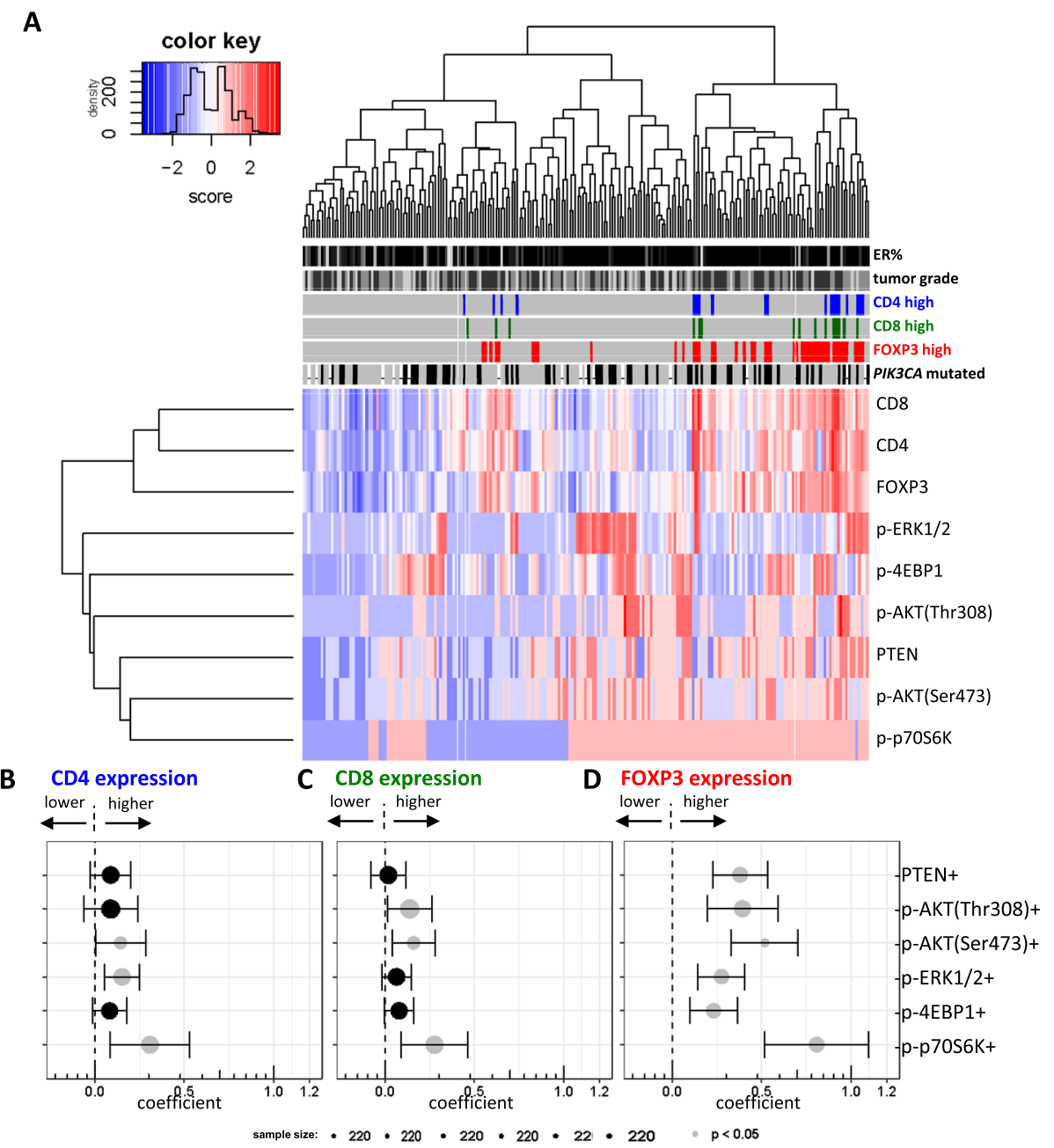

Fig. $\mathbf{5}$ Lymphocytic infiltration and PI3K pathway activation in breast tumors. a Heat map represents unsupervised hierarchical clustering of 215 breast carcinomas (columns) based on the expression of lymphocyte markers and the activation of PI3K pathway on tumor cells (rows). Information on percentage of ER positivity (from 10 to 100\%) and tumor grade $(1,2$ or 3$)$ is indicated by gray scale: low as lighter gray and high as darker gray. Boxes of PIK3CA mutation status are filled with gray (wild-type), black (mutated), or white (unknown). CD4, CD8, and FOXP3 status was filled with color (high) or gray (low). The forest plots represent multivariable linear regression models. Each plot merges 6 multivariable models (described in Additional file 2: Tables S2A-F) calculating the association between the (phospho-) levels of PI3K downstream proteins and CD4 (b), CD8 (c), or FOXP3 (d) expression. Measurements of these associations are represented by the coefficients plotted on the $x$ axes. The size of each dot is proportional to the number of samples used for each multivariable complete case analysis, and bars represent the standard deviation

stroma. Moreover, the dynamic range of FOXP3 expression in the breast tumor microenvironment is limited (0-15\%; illustrated in Additional file 1: Figure S3I). Of note, we also analyzed the CD8/FOXP3 as well as the CD4/FOXP3 ratio and no significant association with survival was observed (data not shown). Further validation of the prognostic value of TIL subsets in luminal breast cancer is required.

The activation of the PI3K pathway plays a central role in cancer growth and tamoxifen resistance [19-21, 23]. Preclinical data suggested that deregulation of the PI3K pathway could contribute to immune escape [43]. On the other hand, detailed information on the cross-talk between the immune infiltrate and cancer cells harboring an aberration in the PI3K pathway in vivo is scarce. Using a large set of breast cancer patients who received adjuvant chemotherapy, Kotoula et al. did not show a clear association between TILs and PIK3CA mutations [44]. In order to investigate the association between the actual activation of the PI3K pathway and immune infiltration, we assessed phosphorylation levels of proteins downstream of PI3K in parallel with TIL subsets defined by CD4, CD8, and FOXP3 [22, 23]. Our analyses of TIL subsets in the context of PI3K alterations revealed that tumors with a PIK3CA mutation tend to have more CD8 cells and tumors enriched for FOXP3-positive cells show downstream activation of the PI3K pathway. Previously, we have shown the lack of clear association between mutations in PIK3CA 
and activation of downstream proteins in the PI3K/AKT/ mTOR pathway [23]. PIK3CA exon 20 mutations are associated with higher p-ERK1/2 levels that belong to the MAP kinases pathway, and tumors with a PIK3CA exon 9 mutation are associated with higher $\mathrm{p}$-AKT and pERK1/2, but not with p-p70S6K [23]. Our current study illustrates that cancer-immune interactions might differ depending on specific alterations in PI3K pathway. Interestingly, our data are in line with Crane et al., who demonstrated using in vitro models that breast carcinoma cells with activated PI3K pathway, once exposed to activated $\mathrm{T}$ cells, adopt an immune-resistant phenotype by increasing the percentage of FOXP3-expressing lymphocytes [43]. More functional studies are needed for a better understanding of the specific interactions between the multifunctional PI3K pathway and the various components of the pro- and anticancer immune response.

We also evaluated the benefit of adjuvant tamoxifen according to the CD8 status of their tumor. Within patients classified as CD8-low, no significant RFI improvement was detected when patients were treated with adjuvant tamoxifen, compared with the control group. Similarly, Blok et al. also observed a limited benefit of adjuvant tamoxifen relative to exemestane within patients with a low number of CD8 TILs [45]. More studies exploring the predictive value of tumor-immune profiles are needed.

Limitations of our study include the relatively small sample size and lack of correction for multiple testing. Furthermore, the patients included in our study did not receive endocrine treatment during $5-10$ years or aromatase inhibitors, according to the current guidelines. Therefore, it remains to be determined whether tumorassociated immune cells are associated with long-term benefit of endocrine treatment. Although we evaluated the PIK3CA-immune interaction in a dataset of a prospective trial, this analysis was not preplanned and should therefore be considered exploratory.

Insufficient data is available on combination treatment with immunotherapy plus PI3K inhibition in luminal breast cancer. Recently, the randomized, phase 3 SOLAR-1 trial showed that the treatment with alpelisib, a PI3K $\alpha$-specific inhibitor, in combination with fulvestrant prolonged progression-free survival among patients with PIK3CAmutated, ER-positive/HER2-negative advanced breast cancer [46]. Given that patients with a PIK3CA mutation in the tumor might have more benefit from PI3K inhibition [24, 25, 47], and, according to our data, could have more tumor-associated CD8, a combination treatment with immune checkpoint blockade plus PI3K inhibition deserves exploration in a clinical trial. Notably, combined PI3K and CDK4/6 inhibition, along with immune checkpoint inhibition induced durable regressions of TN breast cancer tumors in vivo [48]. Based on our observation that PI3K activation is associated with higher levels of regulatory $\mathrm{T}$ cells, the development of PI3K inhibitors in combination with immunomodulatory agents that are able to deplete regulatory $\mathrm{T}$ cells might be of interest for luminal breast cancer [43].

\section{Conclusion}

In this dataset of ER-positive breast cancer, tumor infiltration of CD8-positive lymphocytes was associated with PIK3CA mutations and worse clinical outcome. These associations were more pronounced among patients with grade 1 or 2 tumors. Furthermore, activation of the PI3K pathway, measured using phosphorylated downstream proteins, in breast tumor cells was positively correlated with tumorinfiltrating FOXP3-positive lymphocytes. Further validation of these cancer-immune interactions in ER-positive breast tumors might provide useful information for further development of immunomodulatory combination treatments for ER-positive breast cancers with high TILs.

\section{Additional files}

Additional file 1: Figure S1. Data collection. Source of data and tumor material from the patients entered in the multicenter IKA trial. Figure S2. Validation of the expression values generated by automated fashion. Panel of the comparison between expression evaluated by observers versus expression values obtained from image-analysis software (after normalization). Figure S3. Evaluation of the staining of the lymphocyte markers CD4, CD8 and FOXP3. Figure S4. Distribution of the expression of the lymphocyte markers by tumor characteristics. Figure S5. Distribution of the expression of lymphocyte markers according to PIK3CA mutation status. Figure S6. Analysis of the linearity of the Cox regression functions. Figure S7. Multivariable Cox regression models in ER-positive breast cancer. Figure S8. Association between CD8 status and tamoxifen benefit. Figure S9. Association between CD8 status and tamoxifen benefit within the HER2-negative group. Figure S10. Levels of the percentage of ER positivity are not associated with the status of lymphocyte markers. (PPTX 20084 kb)

Additional file 2: Table S1. Staining details. Table S2. Interaction terms. Table S3. PI3K pathway activation and lymphocytic infiltration. Table S4. Comparison between all patients and those with CD4, CD8, or FOXP3 staining. (PPTX $272 \mathrm{~kb}$ )

\begin{abstract}
Acknowledgements
The authors thank all pathology departments throughout the Netherlands for the submission of formalin-fixed paraffin-embedded tumor blocks. They thank all the individuals who took part in these studies and all the researchers, clinicians, technicians, and administrative staff who have enabled this work to be carried out. Thanks to the Core Facility Molecular Pathology and Biobanking (CFMPB) of the Netherlands Cancer Institute for their technical support and the patients and families who contributed to this study.
\end{abstract}

Authors' contributions

MSL, MKS, SCL, and MK were responsible for the concept and design of the study. MO, IS, JS, DTK, KB, RLPV, EJB, and PJKK contributed substantially to the acquisition of the data. MSL, MKS, MO, IS, DG, VvdN, and MK contributed to the analysis and interpretation of the data. $\mathrm{KVdV}, \mathrm{HMH}, \mathrm{MO}, \mathrm{IS}$, and JS contributed to the data quality control. MSL, with supervision from MKS and MK, drafted the manuscript. All authors critically revised the manuscript for important intellectual content and approved the final version. 


\section{Funding}

This work was supported by grants from TI Pharma (project number T3-502) and from A Sister's Hope. This work was supported by KWF Kankerbestrijding (MKS): https://www.kwf.nl/onderzoek/pages/default.aspx; MSL received a research grant from Conselho Nacional de Desenvolvimento Científico e Tecnológico (CAPES Foundation, Brazil: 99999.001321/2013-07), and MK is a research fellow at the Cancer Immunotherapy of the Dutch Cancer Society. The funders had no role in study design, data collection and analysis, decision to publish, or preparation of the manuscript.

\section{Availability of data and materials}

Data from IKA trial (pathological, clinical, PIK3CA mutation status and PI3K phosphorylation pathways) were discussed in previous publications of the group in Breast Cancer Research [22, 23]. Data is available upon request from the corresponding author.

\section{Ethics approval and consent to participate}

The IKA trial was approved by the central ethics committee of the Netherlands Cancer Institute. Informed consent was obtained from all participating patients. The trial was performed in accordance with the Declaration of Helsinki. For this retrospective translational study, no additional consent was required according to Dutch legislation, since the use of anonymized archival pathology left-over material does not interfere with patient care. Tumor tissue was handled according to the Dutch code of conduct for responsible use of human tissue in the context of health research.

\section{Consent for publication}

The original trial was approved by the central ethics committee of the Netherlands Cancer Institute and informed consent was obtained from all the study participants. For this retrospectively retrospective translational study, no additional consent was required, according to Dutch legislation. AS described in already published papers using data from this trial, (Beelen, $\mathrm{K}$ et al Breast Cancer Research 2014).

\section{Competing interests}

SCL is an advisory board member for AstraZeneca, Cergentis, Novartis, Roche, and Sanofi. SCL received institutional research support funding from Adienne, Amgen, AstraZeneca, Genentech, Roche, Sanofi, and Tesaro. MK is an advisory board member for BMS. MK receives institutional research support from BMS and Roche. The other authors declare that they have no competing interests.

\section{Author details}

${ }^{1}$ Division of Molecular Pathology, Netherlands Cancer Institute, Amsterdam, The Netherlands. ${ }^{2}$ Coordenação de Pesquisa, Instituto Nacional do Câncer, Rio de Janeiro, RJ, Brazil. ${ }^{3}$ Division of Pathology, Netherlands Cancer Institute, Amsterdam, The Netherlands. ${ }^{4}$ Department of Pathology, Netherlands Cancer Institute, Amsterdam, The Netherlands. ${ }^{5}$ Department of Pathology, Ghent University Hospital, Ghent, Belgium. ${ }^{6}$ Department of Medical Oncology, VU University Medical Centre, Amsterdam, The Netherlands. ${ }^{7}$ Department of Biometrics, Netherlands Cancer Institute, Amsterdam, The Netherlands. ${ }^{8}$ Division of Medical Oncology, Reinier de Graaf Hospital, Delft, The Netherlands. ${ }^{9}$ Division of Medical Oncology, Netherlands Cancer Institute, Amsterdam, The Netherlands. ${ }^{10}$ Department of Pathology, University Medical Center Utrecht, Utrecht, The Netherlands. ${ }^{11}$ Department of Surgery, Leiden University Medical Center, Leiden, The Netherlands. ${ }^{12}$ Department of Medical Oncology, Leiden University Medical Centre, Leiden, The Netherlands. ${ }^{13}$ Division of Molecular Oncology and Immunology, Netherlands Cancer Institute, Plesmanlaan 121, 1066 CX Amsterdam, The Netherlands.

\section{Received: 28 January 2019 Accepted: 24 July 2019}

\section{Published online: 07 August 2019}

\section{References}

1. Davies C, Godwin J, Gray R, Clarke M, Cutter D, Darby S, McGale P, Pan HC, Taylor C, Wang YC, et al. Relevance of breast cancer hormone receptors and other factors to the efficacy of adjuvant tamoxifen: patient-level metaanalysis of randomised trials. Lancet. 2011;378(9793):771-84.

2. Davies C, Pan H, Godwin J, Gray R, Arriagada R, Raina V, Abraham M, Medeiros Alencar VH, Badran A, Bonfill X, et al. Long-term effects of continuing adjuvant tamoxifen to 10 years versus stopping at 5 years after diagnosis of oestrogen receptor-positive breast cancer: ATLAS, a randomised trial. Lancet. 2013;381(9869):805-16.

3. Stanton SE, Adams S, Disis ML. Variation in the incidence and magnitude of tumor-infiltrating lymphocytes in breast cancer subtypes: a systematic review. JAMA Oncol. 2016;2(10):1354-60.

4. Savas P, Salgado R, Denkert C, Sotiriou C, Darcy PK, Smyth MJ, Loi S. Clinical relevance of host immunity in breast cancer: from TILS to the clinic. Nat Rev Clin Oncol. 2016;13(4):228-41.

5. Ali HR, Provenzano E, Dawson SJ, Blows FM, Liu B, Shah M, Earl HM, Poole CJ, Hiller L, Dunn JA, et al. Association between CD8+ T-cell infiltration and breast cancer survival in 12,439 patients. Ann Oncol. 2014;25(8):1536-43.

6. Baker K, Lachapelle J, Zlobec I, Bismar TA, Terracciano L, Foulkes WD. Prognostic significance of CD8+ T lymphocytes in breast cancer depends upon both oestrogen receptor status and histological grade. Histopathology. 2011;58(7):1107-16.

7. Denkert C, von Minckwitz G, Darb-Esfahani S, Lederer B, Heppner BI, Weber KE, Budczies J, Huober J, Klauschen F, Furlanetto J, et al. Tumour-infiltrating lymphocytes and prognosis in different subtypes of breast cancer: a pooled analysis of 3771 patients treated with neoadjuvant therapy. Lancet Oncol. 2018;19(1):40-50.

8. Engels CC, Charehbili A, van de Velde CJ, Bastiaannet E, Sajet A, Putter H, van Vliet EA, van Vlierberghe RL, Smit VT, Bartlett JM, et al. The prognostic and predictive value of Tregs and tumor immune subtypes in postmenopausal, hormone receptor-positive breast cancer patients treated with adjuvant endocrine therapy: a Dutch TEAM study analysis. Breast Cancer Res Treat. 2015;149(3):587-96.

9. Liu F, Lang R, Zhao J, Zhang X, Pringle GA, Fan Y, Yin D, Gu F, Yao Z, Fu L. $\mathrm{CD} 8(+)$ cytotoxic $T$ cell and FOXP3(+) regulatory $T$ cell infiltration in relation to breast cancer survival and molecular subtypes. Breast Cancer Res Treat. 2011;130(2):645-55.

10. Liu S, Foulkes WD, Leung S, Gao D, Lau S, Kos Z, Nielsen TO. Prognostic significance of FOXP3+ tumor-infiltrating lymphocytes in breast cancer depends on estrogen receptor and human epidermal growth factor receptor-2 expression status and concurrent cytotoxic T-cell infiltration. Breast Cancer Res. 2014;16(5):432.

11. Mahmoud SM, Paish EC, Powe DG, Macmillan RD, Grainge MJ, Lee AH, Ellis IO, Green AR. Tumor-infiltrating CD8+ lymphocytes predict clinical outcome in breast cancer. J Clin Oncol. 2011;29(15):1949-55.

12. Mahmoud SM, Paish EC, Powe DG, Macmillan RD, Lee AH, Ellis IO, Green AR. An evaluation of the clinical significance of FOXP3+ infiltrating cells in human breast cancer. Breast Cancer Res Treat. 2011;127(1):99-108.

13. Shang B, Liu Y, Jiang SJ, Liu Y. Prognostic value of tumor-infiltrating FoxP3+ regulatory $T$ cells in cancers: a systematic review and meta-analysis. Sci Rep. 2015;5:15179.

14. Kortlever RM, Sodir NM, Wilson CH, Burkhart DL, Pellegrinet L, Brown Swigart L, Littlewood TD, Evan Gl. Myc cooperates with Ras by programming inflammation and immune suppression. Cell. 2017;171(6): $1301-15$ e1314.

15. Layer JP, Kronmuller MT, Quast T, van den Boorn-Konijnenberg D, Effern M, Hinze D, Althoff K, Schramm A, Westermann F, Peifer M, et al. Amplification of N-Myc is associated with a T-cell-poor microenvironment in metastatic neuroblastoma restraining interferon pathway activity and chemokine expression. Oncoimmunology. 2017;6(6):e1320626.

16. Quigley D, Silwal-Pandit L, Dannenfelser R, Langerod A, Vollan HK, Vaske C, Siegel JU, Troyanskaya O, Chin SF, Caldas C, et al. Lymphocyte invasion in IC10/Basal-Like breast tumors is associated with wild-type TP53. Mol Cancer Res. 2015;13(3):493-501.

17. Rooney MS, Shukla SA, Wu CJ, Getz G, Hacohen N. Molecular and genetic properties of tumors associated with local immune cytolytic activity. Cell. 2015;160(1-2):48-61.

18. Shen Q, Cohen B, Zheng W, Rahbar R, Martin B, Murakami K, Lamorte S, Thompson P, Berman H, Zuniga-Pflucker JC, et al. Notch shapes the innate immunophenotype in breast cancer. Cancer Discovery. 2017;7(11):1320-35.

19. Guerrero-Zotano A, Mayer IA, Arteaga CL. PI3K/AKT/mTOR: role in breast cancer progression, drug resistance, and treatment. Cancer Metastasis Rev. 2016;35(4):515-24.

20. Azim HA, Kassem L, Treilleux I, Wang Q, El Enein MA, Anis SE, Bachelot T. Analysis of PI3K/mTOR pathway biomarkers and their prognostic value in women with hormone receptor-positive, HER2-negative early breast cancer. Transl Oncol. 2016;9(2):114-23. 
21. Yang SX, Polley E, Lipkowitz S. New insights on PI3K/AKT pathway alterations and clinical outcomes in breast cancer. Cancer Treat Rev. 2016;45:87-96.

22. Beelen K, Opdam M, Severson TM, Koornstra RH, Vincent AD, Wesseling J, Muris JJ, Berns EM, Vermorken JB, van Diest PJ, et al. Phosphorylated p70S6K predicts tamoxifen resistance in postmenopausal breast cancer patients randomized between adjuvant tamoxifen versus no systemic treatment. Breast Cancer Res. 2014;16(1):R6.

23. Beelen K, Opdam M, Severson TM, Koornstra RH, Vincent AD, Wesseling J, Muris JJ, Berns EM, Vermorken JB, van Diest PJ, et al. PIK3CA mutations, phosphatase and tensin homolog, human epidermal growth factor receptor 2, and insulin-like growth factor 1 receptor and adjuvant tamoxifen resistance in postmenopausal breast cancer patients. Breast Cancer Res. 2014;16(1):R13.

24. Baselga J, Im SA, Iwata H, Cortes J, De Laurentiis M, Jiang Z, Arteaga CL, Jonat W, Clemons M, Ito Y, et al. Buparlisib plus fulvestrant versus placebo plus fulvestrant in postmenopausal, hormone receptor-positive, HER2negative, advanced breast cancer (BELLE-2): a randomised, double-blind, placebo-controlled, phase 3 trial. Lancet Oncol. 2017;18(7):904-16.

25. Andre F, Campone M, Ciruelos EM, Iwata H, Loibl S, Rugo HS, Wilke C, Mills D, Chol M, Longin A-S, et al. SOLAR-1: a phase III study of alpelisib + fulvestrant in men and postmenopausal women with HR+/HER2- advanced breast cancer $(\mathrm{BC})$ progressing on or after prior aromatase inhibitor therapy. J Clin Oncol. 2016:34(15 suppl):TPS618.

26. Rugo HS, Delord JP, Im SA, Ott PA, Piha-Paul SA, Bedard PL, Sachdev J, Tourneau CL, van Brummelen EMJ, Varga A, et al. Safety and antitumor activity of pembrolizumab in patients with estrogen receptor-positive/ human epidermal growth factor receptor 2-negative advanced breast cancer. Clin Cancer Res. 2018;24(12):2804-11.

27. Craddock CG, Longmire R, McMillan R. Lymphocytes and the immune response. 1. N Engl J Med. 1971;285(6):324-31.

28. Antony PA, Piccirillo CA, Akpinarli A, Finkelstein SE, Speiss PJ, Surman DR, Palmer DC, Chan CC, Klebanoff CA, Overwijk WW, et al. CD8+ T cell immunity against a tumor/self-antigen is augmented by CD4+ T helper cells and hindered by naturally occurring T regulatory cells. J Immunol. 2005;174(5):2591-601.

29. Schreiber RD, Old LJ, Smyth MJ. Cancer immunoediting: integrating immunity's roles in cancer suppression and promotion. Science. 2011; 331(6024):1565-70

30. Marie JC, Letterio JJ, Gavin M, Rudensky AY. TGF-beta1 maintains suppressor function and Foxp3 expression in CD4+CD25+ regulatory T cells. J Exp Med. 2005;201(7):1061-7.

31. Ghebeh H, Barhoush E, Tulbah A, Elkum N, Al-Tweigeri T, Dermime S. FOXP3+ Tregs and B7-H1+/PD-1+ T lymphocytes co-infiltrate the tumor tissues of high-risk breast cancer patients: implication for immunotherapy. BMC Cancer. 2008;8:57.

32. Vermorken JB, Burgers JMV, Taat CW, van de Slee PHT, Hennipman A, Norman JWR, Rozendaal KJ, van Tinteren H, Huldij J, Benraadt J. Adjuvant tamoxifen in breast cancer: interim results of a comprehensive cancer center Amsterdam trial. Breast Cancer Res Treat. 1998;50(abstracts poster session III):329.

33. Beelen K, Opdam M, Severson T, Koornstra R, Vincent A, Wesseling J, Sanders J, Vermorken J, van Diest P, Linn S. Mitotic count can predict tamoxifen benefit in postmenopausal breast cancer patients while Ki67 score cannot. BMC Cancer. 2018;18(1):761.

34. Kruger DT, Beelen KJ, Opdam M, Sanders J, van der Noort V, Boven E, Linn SC. Hierarchical clustering of activated proteins in the PI3K and MAPK pathways in ER-positive, HER2-negative breast cancer with potential therapeutic consequences. Br J Cancer. 2018;119(7):832-9.

35. Forrest R, Guthrie GJ, Orange C, Horgan PG, McMillan DC, Roxburgh CS. Comparison of visual and automated assessment of tumour inflammatory infiltrates in patients with colorectal cancer. Eur J Cancer. 2014:50(3):544-52.

36. Bartko JJ. The intraclass correlation coefficient as a measure of reliability. Psychol Rep. 1966;19(1):3-11.

37. Roshani D, Ghaderi E. Comparing smoothing techniques for fitting the nonlinear effect of covariate in Cox models. Acta Informatica Medica. 2016; 24(1):38-41.

38. Vermont J, Bosson JL, Francois P, Robert C, Rueff A, Demongeot J. Strategies for graphical threshold determination. Comput Methods Prog Biomed. 1991;35(2):141-50.

39. Kraemer HC. Correlation coefficients in medical research: from product moment correlation to the odds ratio. Stat Methods Med Res. 2006;15(6): $525-45$.
40. Cohen J. A coefficient of agreement for nominal scales. Educ Psychol Meas. 1960;20(1):37-46.

41. Papaxoinis G, Kotoula V, Alexopoulou Z, Kalogeras KT, Zagouri F, Timotheadou E, Gogas H, Pentheroudakis G, Christodoulou C, Koutras A, et al. Significance of PIK3CA mutations in patients with early breast cancer treated with adjuvant chemotherapy: a Hellenic Cooperative Oncology Group (HeCOG) study. PLoS One. 2015;10(10):e0140293.

42. Huang JZ, Shen H. Functional coefficient regression models for non-linear time series: a polynomial spline approach. Scand J Stat. 2004;31(4):515-34.

43. Crane CA, Panner A, Murray JC, Wilson SP, Xu H, Chen L, Simko JP, Waldman FM, Pieper RO, Parsa AT. PI(3) kinase is associated with a mechanism of immunoresistance in breast and prostate cancer. Oncogene. 2009;28(2):306-12

44. Kotoula V, Karavasilis V, Zagouri F, Kouvatseas G, Giannoulatou E, Gogas H, Lakis S, Pentheroudakis G, Bobos M, Papadopoulou K, et al. Effects of TP53 and PIK3CA mutations in early breast cancer: a matter of co-mutation and tumor-infiltrating lymphocytes. Breast Cancer Res Treat. 2016;158(2):307-21.

45. Blok EJ, Engels CC, Dekker-Ensink G, Meershoek-Klein Kranenbarg E, Putter $\mathrm{H}$, Smit V, Liefers GJ, Morden JP, Bliss JM, Coombes RC, et al. Exploration of tumour-infiltrating lymphocytes as a predictive biomarker for adjuvant endocrine therapy in early breast cancer. Breast Cancer Res Treat. 2018; 171(1):65-74.

46. Andre F, Ciruelos E, Rubovszky G, Campone M, Loibl S, Rugo HS, Iwata H, Conte P, Mayer IA, Kaufman B, et al. Alpelisib for PIK3CA-mutated, hormone receptor-positive advanced breast cancer. N Engl J Med. 2019;380(20):1929-40.

47. Di Leo A, Johnston $\mathrm{S}$, Lee KS, Ciruelos E, Lonning PE, Janni W, O'Regan R, Mouret-Reynier MA, Kalev D, Egle D, et al. Buparlisib plus fulvestrant in postmenopausal women with hormone-receptor-positive, HER2-negative, advanced breast cancer progressing on or after mTOR inhibition (BELLE-3): a randomised, double-blind, placebo-controlled, phase 3 trial. Lancet Oncol. 2018;19(1):87-100

48. Teo ZL, Versaci S, Dushyanthen S, Caramia F, Savas P, Mintoff CP, Zethoven M, Virassamy B, Luen SJ, McArthur GA, et al. Combined CDK4/6 and PI3Kalpha inhibition is synergistic and immunogenic in triple-negative breast cancer. Cancer Res. 2017;77(22):6340-52.

\section{Publisher's Note}

Springer Nature remains neutral with regard to jurisdictional claims in published maps and institutional affiliations.

\section{Ready to submit your research? Choose BMC and benefit from:}

- fast, convenient online submission

- thorough peer review by experienced researchers in your field

- rapid publication on acceptance

- support for research data, including large and complex data types

- gold Open Access which fosters wider collaboration and increased citations

- maximum visibility for your research: over $100 \mathrm{M}$ website views per year

At $\mathrm{BMC}$, research is always in progress.

Learn more biomedcentral.com/submission 